\title{
Is periodontitis associated with raised blood pressure or hypertension? Evidence from the National Health and Nutrition Examination Survey (NHANES), 2001-2002
}

\author{
Jing Guan ${ }^{1 *}$, Jian-Long Zhai ${ }^{2}$, Hao-Gong Wang ${ }^{3}$ and Chao Liu ${ }^{4}$ \\ ${ }^{1}$ Stomatology Division, Hebei General Hospital, Shijiazhuang, China \\ ${ }^{2}$ Heart Center, Hebei General Hospital, Shijiazhuang, China \\ ${ }^{3}$ Stomatology Hospital, Hebei Medical university, Shijiazhuang, China \\ ${ }^{4}$ The First Cardiology Division, The First Hospital of Hebei Medical University, Shijiazhuang, China
}

Submission: September 17, 2020; Published: October 19, 2020

*Corresponding author: Jing Guan, Stomatology Division, Hebei General Hospital, 348 Heping west Road, Shijiazhuang, Hebei Province, China

\section{Abstract}

Introduction: There is incomplete and inconclusive evidence for the correlation between periodontitis and blood pressure, particularly from large national epidemiological studies. The aim of this cross-sectional study was to assess the relationship between periodontitis and blood pressure and hypertension in people in USA, and to analyze confounding factors in both of them.

Materials and Methods: We analyzed data from the National Health and Nutrition Examination Survey (NHANES) from 2001 to 2002 on 6617 men and 7377 women who received a periodontal examination and a blood pressure measurement. We analyzed blood pressure in both a continuous format and a binary variable for case definition of hypertension. Periodontitis was defined by clinical measures of attachment level (AL), probing depth (PD), and probing bleeding (PB), which estimated further the severity of periodontitis. Relationships between periodontitis and blood pressure were analyzed before and after adjustment for confounding factors.

Results: Gender difference was found between systolic blood pressure (SBP) and diastolic blood pressure (DBP), hypertension and nonhypertension, periodontitis and non-periodontitis. The prevalence of hypertension and periodontitis varied among different age groups. There was a slight link between blood pressure and periodontitis before and after adjusting for gender and age.

Conclusion: We did observe the relationship between periodontitis and blood pressure. We also found that some other risk factors, such as gender and age, influenced the development and progress of both blood pressure and periodontitis.

Keywords: Blood pressure; Epidemiology; Health survey; Hypertension; Periodontitis

\section{Introduction}

Periodontitis is a highly-prevalent chronic inflammatory condition of the tissues that affects the supporting structures of the teeth, including the periodontal ligament, cementum and the alveolar bone [1,2]. It is featured with a chronic compounded infection, which is caused by several microbial agents [3]. Periodontal disease is a chronic infection that has been documented to insult over $50 \%$ of the general population [3]. Severe generalized periodontitis affects $5 \%$ to $15 \%$ of any population worldwide and is a major cause of tooth loss [4]. If left untreated, it leads to deterioration of the supportive tissue of the teeth and eventually to tooth loss. Nevertheless, periodontitis seemingly affects more than just the supporting tissues of the teeth.

Hypertension is the most prevalent of all cardiovascular diseases (CVDs) affecting about $30-40 \%$ of adults [5], and is one of the main issues of hospitalization and morbidity worldwide. Hypertension is considered as a condition of dynamic interaction between diverse genetic, physiological, environmental and 
psychosocial factors [6]. In addition, hypertension seems to be involved in $50 \%$ of the deaths due to CVDs [5]. It is a strong risk factor for stroke and CVDs, and is closely associated with endothelial dysfunction [7], and along with other factors promotes the atherosclerotic process. In addition to many dietary and lifestyle factors including smoking and alcohol consumption [8], chronic inflammation has played a role in the etiology of hypertension [9].

To date, the evidence for the association between periodontitis and blood pressure is incomplete and inconclusive, especially from large national epidemiological studies. Even after adjusting for the effect of socio-demographic, behavioral and physiological factors, and chronic diseases, gingival bleeding was consistently and significantly associated with raised SBP and an higher prevalence of hypertension in the US adult population [10]. This crosssectional study assessed the relationship between periodontitis with arterial blood pressure in people aged 15 years and over in USA. We analyzed data from NHANES from 2001 to 2002 on 2664 men and 2774 women who received both a periodontal examination and conventional blood pressure measurements. Blood pressure was analyzed in both a continuous format and a binary variable for case definition of hypertension. To definite periodontitis and its severity, we use the case definitions for periodontitis developed by the Centers for Disease Control and Prevention (CDC) and the American Academy of Periodontology (AAP) in 2003 [11]. The aim of this study was to investigate the relationship between periodontitis and its severity with blood pressure and hypertension. Periodontitis significantly related with SBP and DBP, even after adjusting for the effect of major confounding factors, for instance, gender, age, race, and marital status. In the long run, this relationship might play a positive role in clinical practice and public health strategies.

\section{Materials and Methods}

In this study, we evaluated the association between periodontitis and hypertension and blood pressure, and potential confounding factors about periodontitis and blood pressure were also analyzed. Data for this study was from NHANES from 2001 to 2002, supported by United States Department of Health and Human Services, Centers for Disease Control and Prevention, and National Center for Health Statistics.

\section{Data Collection}

Examination Data was collected by the Mobile Examination Center (MEC). The qualifications for MEC laboratory, physician, dental, and health technician staff were described in the component training manuals. All MEC staff completed the requirements for safety, subject privacy and confidentiality, and cardiopulmonary resuscitation training. In addition, all staff completed component specific training to learn the standardized NHANES protocol for each of the components they performed. The health technicians, for example, performed multiple procedures and were expected to maintain their skill levels on each component.

\section{Quality Control during Data Collection}

The quality of data in this survey is controlled by an intense training period for the dental teams with calibration of dental examiners prior to the beginning of the survey, periodic monitoring and recalibration of dental examiners, and periodic retraining of dental teams. The reference examiner will visit each team three times per year to observe field operations and to replicate 20-25 dental examinations during each visit. The purpose of these "expert replications" is to determine if the field examiners are maintaining the examination standards achieved during training and to measure the degree of deviation, if any, from those standards. If the inter-rater correlation is not within acceptable limits, retraining is conducted on site and future monitoring of the field examiner intensified. An annual retraining session for each dental examiner also is conducted by the reference examiner to reinforce existing protocols and to accommodate protocol updates as needed. Approximately $10 \%$ of examined participants are asked to return for a replicate exam. The purpose of these "repeat exams" is to monitor internal consistency within examiners regarding the data collection process.

\section{Survey Staff}

The oral health exam is performed by trained dentists who hold a state dental license in a U.S. jurisdiction. One of up to seven trained oral health recorders is paired with a dentist to form a dental examination team. Two teams function independently throughout the data collection period. All oral health exam data are collected in the mobile examination center. Oral health data obtained during the home interview are collected by trained interviewers. Interview and examination protocols are discussed in detail in the NHANES Oral Health Home Interview and Exam Training Manuals.

\section{Sample Selection}

Participants aged 2 years and older who do not meet any of the exclusion criteria are eligible. A positive response to any of the following medical health-screening questions asked of participants aged 13 years or older will exclude them from the periodontal and root caries assessments. Participants aged 13-15 years must have a health proxy signed by a parent/guardian to participate in the oral health examination. The medical healthscreening questions are matched as follow: 1) Has a doctor or dentist ever told you that you must always take antibiotics (e.g. penicillin) before you geta dental checkup or care? 2) Do you have a heart problem (specifically, congenital heart murmurs, heart valve problems, congenital heart disease, or bacterial endocarditis)? 3) Do you have rheumatic fever? 4) Kidney disease requiring renal dialysis? 5) Hemophilia? 6) Pacemaker or automatic defibrillator or artificial material in your heart veins or arteries?

\section{Hypertension Assessment}

Blood pressure of the upper arm was measured by trained and calibrated physicians using a mercury sphygmomanometer 
according to standardized BP measurement protocols [12]. The subject was asked to sit down for at least 5 min before blood pressure was measured. The measurement was repeated twice, and an interval of 2 minutes between each time. A third or fourth measure was taken if blood pressure values had great variation. Three or four readings for the SBP and DBP were taken in the examination centre and the measurement referred to the average of these readings. Hypertension was defined by SBP $\geq 140 \mathrm{mmHg}$ or DBP $\geq 90 \mathrm{mmHg}$.

\section{Periodontal Examination}

Periodontal measures were assessed on randomly assigned half-mouths, one upper quadrant and one lower quadrant selected at the beginning of the examination. For periodontitis, clinical measures of $\mathrm{AL}, \mathrm{PD}$, and $\mathrm{PB}$ were obtained. Three interproximal sites were used (mid-facial, mesio-facial, and distal) to determine disease status; AL and PD were rounded up to the nearest millimeter. Severe periodontitis was defined as the presence of 2 or more interproximal sites with $\geq 6 \mathrm{~mm} \mathrm{AL}$ (not on the same tooth) and 1 or more interproximal site(s) with $\geq 5 \mathrm{~mm}$ PD. Moderate periodontitis was defined as 2 or more interproximal sites with $\geq 4 \mathrm{~mm}$ clinical AL (not on the same tooth) or 2 or more interproximal sites with $\mathrm{PD} \geq 5 \mathrm{~mm}$, also not on the same tooth. Mild periodontitis was defined as $\geq 2$ interproximal sites with $\geq$ $3 \mathrm{~mm}$ AL and $\geq 2$ interproximal sites with $\geq 4 \mathrm{~mm}$ PD (not on the same tooth) or 1 site with $\geq 5 \mathrm{~mm}$. Total periodontitis was the sum of severe, moderate, and mild periodontitis [11] (Table 1). Non-periodontitis was defined as the conditions except all above.

Table 1: The definition and severity of periodontitis case.

\begin{tabular}{|c|c|c|c|c|c|}
\hline & \multicolumn{2}{|c|}{$\mathrm{AL}^{\mathrm{a}}$ (not on the same tooth) } & & \multicolumn{2}{|c|}{$P^{b}$ (not on the same tooth, except for severe case) } \\
\hline & Depth $(\mathrm{mm})$ & Site & & Depth $(\mathrm{mm})$ & Site \\
\hline Non & $<3$ & & Or & $<4$ & $<2$ \\
\hline \multirow{2}{*}{ Mild } & \multirow{2}{*}{$\geq 3$} & \multirow{2}{*}{$\geq 2$} & \multirow{2}{*}{ And } & $\geq 4$ & $\geq 2$ \\
\hline & & & & $\geq 5$ & $\geq 1$ \\
\hline Moderate & $\geq 4$ & $\geq 2$ & Or & $\geq 5$ & $\geq 2$ \\
\hline Severe & $\geq 6$ & $\geq 2$ & And & $\geq 5$ & $\geq 1$ \\
\hline
\end{tabular}

${ }^{a}$ Attachment level. ${ }^{b}$ Probing depth.

\section{Potential Confounding Factors and Other Factors}

This survey also collected possible confounders in relation to periodontitis and blood pressure, as well as socioeconomic and demographic background. This included variables on gender, age, race, military status, country of birth, citizenship status, annual household income, annual family income, pregnancy status, weight, heart rate, pulse type, and each reading and average value of SBP and DBP.

\section{Statistical Analyses}

All statistical analyses were carried out using Statistical Package for Social Sciences (SPSS 23.0) with a significance level of $5 \%(\mathrm{P}<0.05)$. Age, heart rate, total number of people in the household,AL, PD, BOP, SBP, andDBP were expressed by means and standard deviation, and comparisons between groups performed using Student's t test. Gender, race, military status, country of birth, citizenship status, education level, marital status, annual household income, annual family income, pulse type were presented by frequency distribution, and comparisons between groups performed using chi-squared test. Linear regression models were used for both SBP and DBP measured continuously and logistic regression models for HBP. Two models of logistic regression test were performed to assess the association of age with hypertension and periodontitis. Any statistically significant association was also confirmed in the subgroup of participants.
Subgroup analyses were performed by sex and age. From the initial sample of 10478 dentate participants, periodontal examination was not carried out on 3348 participants. Furthermore, 495 participants had missing data in relation to other variables used in the analysis; therefore regression analyses models were based on 6635 dentate participants.

\section{Results}

The total sample comprised 7130 participants. The average SBP and DBP were not obtained from 359 participants. 1279 participants did not complete periodontal measurement. Hence, 5492 effective values were finally analyzed.

\section{Analysis of Blood Pressure}

The average SBP was 120.2 (95\% confidence interval 119.4, 120.9) $\mathrm{mmHg}$ for men and $116.2(115.4,116.9) \mathrm{mmHg}$ for women. The respective figures for DBP were $69.6(69.1,70.1) \mathrm{mmHg}$ for men and $67.5(67.1,67.9) \mathrm{mmHg}$ for women (Table 2). When we used independent-samples $\underline{t}$ test to analyze influence factors of blood pressure, there were also statistical differences in the mean SBP and DBP by military status, citizenship status, annual household income, pregnancy status, and ethnicity. Furthermore, compared with participants who smoked in the past 30 minutes, those who did not smoke had lower SBP. Participants with regular pulses had lower SBP than irregular ones. There were statistical 
significances for SBP and DBP among different races, education levels, marital status, and age groups (younger than 40-year, 40-year to 60-year, older than 60-year) (Table 3). In different pulse groups (less than 60,60 to 100 , more than 100), there were differences in SBP, but not in DBP. In the unadjusted linear regression models referring to the whole population, age had statistically significant association with SBP and DBP.

Table 2: Weighted distribution of blood pressure (BP) and periodontitis by gender and age.

\begin{tabular}{|c|c|c|c|c|c|}
\hline & \multicolumn{2}{|c|}{ Gender } & \multicolumn{3}{|c|}{ Age group } \\
\hline & Male $\left(n=2645^{a}\right)$ & Women $\left(n=2789^{a}\right)$ & Yong-age $\left(n=3379^{a}\right)$ & $\operatorname{Mid-age}\left(n=1195^{a}\right)$ & Eld-age $\left(n=814^{a}\right)$ \\
\hline Average SBP(mmHg) & $120.2(119.6-120.9)$ & $116.2(115.4-116.9)$ & 111.1(110.8-111.5) & $123.6(122.6-124.6)$ & $\begin{array}{c}137.4(135.9- \\
138.9)\end{array}$ \\
\hline $\begin{array}{l}\text { Average DB- } \\
\text { P(mmHg) }\end{array}$ & $69.6(69.1-70.1)$ & $67.5(67.1-67.9)$ & $65.1(64.7-65.5)$ & $76.6(76.0-77.2)$ & 71.1(70.2-71.9) \\
\hline Hypertension $^{\mathrm{b}}(\%)$ & $23.0(21.5-24.5)$ & $22.2(20.8-23.7)$ & $5.3(4.6-6.1)$ & $32.9(30.5-35.4)$ & $58.2(55.3-61.0)$ \\
\hline Periodontitisc(\%) & $21.0(19.5-22.4)$ & $15.7(14.5-17.0)$ & 9.1(8.1-10.0) & $25.3(23.0-27.5)$ & $35.7(33.0-38.5)$ \\
\hline Non $(\%)$ & $79.0(77.6-80.5)$ & $84.3(83.0-85.5)$ & $90.9(90.0-91.9)$ & $74.7(72.5-77.0)$ & $64.3(61.5-67.0)$ \\
\hline Mild (\%) & $4.3(3.6-5.0)$ & $3.7(3.0-4.3)$ & $2.9(2.3-3.4)$ & $7.0(5.7-8.3)$ & $3.8(2.7-4.9)$ \\
\hline Moderate (\%) & $14.4(13.1-15.6)$ & $10.8(9.7-11.9)$ & $5.5(4.8-6.3)$ & 15.8(13.9-17.7) & $27.7(25.1-30.3)$ \\
\hline Severe (\%) & $2.3(1.8-2.8)$ & $1.2(0.9-1.6)$ & $0.7(0.4-1.0)$ & $2.5(1.6-3.3)$ & $4.3(3.1-5.4)$ \\
\hline
\end{tabular}

aWeighted number of persons with available data. 'Definition of hypertension, see the text. 'Periodontitis variable refers to percentages with nomild, moderate and severe periodontitis (for definitions of diagnoses, see the text).

Table 3: Other significant risk factors of hypertension and periodontitis.

\begin{tabular}{|c|c|c|c|c|c|c|}
\hline & \multicolumn{2}{|c|}{ Military status } & \multicolumn{2}{|c|}{ Pregnancy status } & \multicolumn{2}{|c|}{ Marital status } \\
\hline & Veteran $(n=648 a)$ & $\begin{array}{l}\text { Non-veter- } \\
\text { an(n=4357a) }\end{array}$ & $\begin{array}{c}\text { Pregnan- } \\
\text { cy(n=305a) }\end{array}$ & $\begin{array}{l}\text { Non-pregnan- } \\
\text { cy }(n=2134 a)\end{array}$ & $\operatorname{Married}(\mathrm{n}=2580 \mathrm{a})$ & Otherd(n=3204a) \\
\hline $\begin{array}{c}\text { Average SB- } \\
\text { P(mmHg) }\end{array}$ & $132.9(131.4-134.5)$ & $123.8(123.1-124.4)$ & $\begin{array}{c}107.6(106.4- \\
108.7)\end{array}$ & $114.3(113.6-115.0)$ & $126.5(125.7-127.3)$ & $119.8(119.1-120.5)$ \\
\hline $\begin{array}{l}\text { Average DB- } \\
\text { P(mmHg) }\end{array}$ & $74.4(73.4-75.4)$ & $71.2(70.8-71.6)$ & $59.6(58.2-60.9)$ & $69.1(68.6-69.6)$ & $73.3(72.8-73.7)$ & $68.0(67.5-68.5)$ \\
\hline Hypertension ${ }^{\mathrm{b}}(\%)$ & $42.1 \quad(38.3-45.9)$ & $24.9(23.6-26.2)$ & $1.3(0.0-2.5)$ & $12.1(10.7-13.4)$ & $31.2(29.4-32.9)$ & $17.5(16.2-18.8)$ \\
\hline Periodontitis ${ }^{c}(\%)$ & $31.8(28.2-35.3)$ & $19.4(18.2-20.6)$ & $10.8(7.3-14.2)$ & $11.7(10.3-13.1)$ & $22.8(21.2-24.4)$ & $15.8(14.6-17.1)$ \\
\hline
\end{tabular}

aWeighted number of persons with available data. 'Definition of hypertension, see the text. 'Periodontitis variable refers to percentages with no-

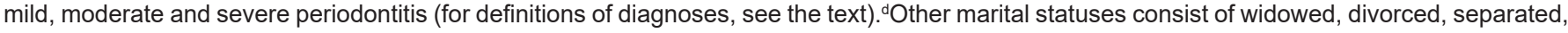
never married, living with partner.

\section{Analysis of Periodontitis}

According to the aforementioned case definition of periodontitis, the total sample consisted of 4595 non-periodontitis participants, 213 mild periodontitis participants, 598 moderate periodontitis participants, 86 severe periodontitis participants. Male group had higher prevalence of periodontitis than female group (Table 2). The same difference was between no/mild periodontitis group and moderate/severe periodontitis group. The prevalence of periodontitis were different among different age groups. Before adjustment, there was a weak positive linear association between age and periodontitis prevalence. The same association was between no/mild periodontitis group and moderate/severe periodontitis group. In all age groups, the prevalence of moderate periodontitis was higher than that of mild and severe periodontitis. Veteran group had higher prevalence of periodontitis than non-veteran group. The prevalence of periodontitis differed among different marital status groups, and married group was obviously higher than other marital groups. However, widowed and divorced groups were obviously higher than other groups. There was no difference in periodontitis prevalence between pregnancy group and non-pregnancy group (Table 3).

\section{Association of Blood Pressure and Periodontitis}

Participants with periodontitis had higher SBP by $8.97(95 \%$ CI: 7.54-10.41) and higher DBP 3.96 (95\% CI: 3.04-4.88) mmHg. Furthermore, compared to no or mild periodontitis participants, moderate or severe periodontitis participants had higher SBP by 10.17 (95\% CI: 8.49-11.85) and higher DBP 3.66 (95\% CI: 2.614.71) $\mathrm{mmHg}$. Participants with periodontitis had higher prevalence of hypertension than those with non-periodontitis (OR=2.91; 95\% CI: 2.54-3.35). The same difference was observed between no/mild periodontitis group and moderate/severe periodontitis group (OR=3.34; 95\% CI: 2.88-3.89). According to aforementioned analysis, we concluded that the important common factors of 
blood pressure and periodontitis were age, and gender. After adjusted for age and gender, we observed a significant difference between periodontitis and non-periodontitis (RR=1.46; 95\% CI: 1.24-1.71). The same difference was observed between no/mild periodontitis and moderate/ severe periodontitis ( $R R=1.56$; 95\% CI: 1.30-1.86). However, after dividing the subjects into three age groups $(<40,40$ to 60 , and $>60$ years $)$ and correcting for gender and age, a significant difference about prevalence of hypertension was only seen in the middle-aged group (40 to 60 years). Introducing age, sex, and pulse times as possible effect modifiers did not result in a significantly better regression model, or in significant regression coefficients of these effect modifiers.

\section{Discussion}

Up to date, though restricted evidence, there has been generally but not completely supportive of an existing association between periodontitis, BP and possibly hypertension $[10,13]$. The present study also show the similar relationship between periodontitis, blood pressure, and hypertension.

The results of this study showed that there was a relationship between hypertension with gender and age. Male participants had higher SBP and DBP than female participants. However, adjusted for age, we found that the prevalence of hypertension of male was higher than female in low age group ( $<40$ years); the prevalence of hypertension of male was lower than female in high age group $(>60$ years); and there was no significant difference between male and female about hypertension prevalence in middle age group. This phenomenon was in accordance with Dubey's findings [14]. They found that gender has an important influence on blood pressure, with premenopausal women having a lower arterial blood pressure than age-matched men. Compared with premenopausal women, postmenopausal women have higher blood pressure. Sex hormones may modulate blood pressure. There are also some social and behavior factors that might result in difference of blood pressure between male and female, but effective evidences are quite limited. The prevalence of hypertension differed among different age groups, and was positively related with age. The cause of hypertension in older patients is incompletely clear, however, there are several pathophysiologic factors as follows: lifestyles, increased relative central adiposity with aging, atherosclerosis and other aging effects on the carotid and aortic baroreceptor sensitivity, impaired ability to excrete sodium, decreased vascular beta-adrenergic vasodilatation function, impaired endotheliummediated vasorelaxation, increased rigidity and decreased compliance of large vessels, and hyaline degeneration within media of precapillary arterioles [15].

Our study also showed other possible factors, such as military status, citizenship status, annual household income, pregnancy status, and ethnicity. Some changes occur in the early period of pregnancy, including increased maternal plasma volume, cardiac output and heart rate, and decreased maternal systemic vascular, resistance and arterial blood pressure [16]. These changes are due to stimulation of the renin-angiotensin-aldosterone system [17]. In addition, the structural changes of maternal cardiovascular system also occur during pregnancy [18]. Therefore, pregnancy participants had higher prevalence of hypertension than nonpregnancy ones, just as what we observed. As for other factors, we need more evidence to explain.

The results of our study showed that the prevalence of periodontitis of male had higher than female, and the same difference was between no/mild periodontitis and moderate/ severe periodontitis. Women matched for body shape with men had worsened periodontal measures and inflammation, however, these women did not lose more teeth [19]. Another study showed that due to adiposity and subclinical inflammation, men had higher prevalence of tooth loss than women. After adjusting for demographics, socioeconomic status, and oral health behaviors, type 2 diabetes was significantly related with moderate-severe periodontitis in male, but not in female. Body mass index and the use of mouthwash were possible reasons for the sex difference. However, a review of the results of various studies showed that the prevalence of aggressive periodontitis in most populations was similar in gender groups, and only a few studies reported gender differences in disease prevalence [20].

In our study, the prevalence of periodontitis varied and was related linearly with age. Mean AL increased linearly with age, but mean PD was constant across age groups in both populations [21]. An early research concluded that when good oral hygiene was maintained, the effect of age on the progression of periodontitis was considered negligible [22]. According to the National Health and Nutrition Examination Surveys (NHANES) 1999-2004, Jung Ki Kim found that African Americans had a faster progression of oral health problems with age [23]. On account of common confounding factors, it is difficult to estimate the relationship of periodontitis and age.

The results of our study showed that prevalence of periodontitis varied with military status, marital status, but not with pregnancy status. Age greater than 30 , smoking, male sex, and Filipino racial background were risk indicators for the presence of moderate or advanced periodontitis, and possibly resulted in higher periodontitis prevalence in military participants [24]. Marital status has a limited impact on periodontal health, but may have a greater impact on several systemic conditions, especially in widowed, divorced, or never married women [25]. Our study also found that compared to other marital status, widowed and divorced groups had significantly higher prevalence of periodontitis. However, married group also had higher prevalence of periodontitis than other marital groups, which was further needed to analyze. Compared with pregnancy, the prevalence of periodontitis decreased from $66.7 \%$ to $33.3 \%$ after delivery. Pregnancy may be related with an increased risk of periodontal disease, but not in accordance with our study. 
In the present study, participants of periodontitis had higher SBP and DBP, and had higher prevalence of hypertension, even after adjusted for age and gender. Evidence up to date though restricted has been generally but not globally supportive of an existing association between periodontitis, BP and possibly hypertension. A recent large study was performed on data derived from the 3rd National Health and Nutrition Examination Survey (NHANES III) on almost 12,000 adult dentate participants to examine associations between periodontal disease measures and BP levels, by applying an extensive adjustment process [17]. Mean SBP was reported higher among participants with moderate or severe periodontitis compared to mild disease, in almost all age groups. A positive linear relationship between SBP and increased severity of periodontitis was further identified in middle-aged subjects. After adjusting for age, sex and ethnicity, a 10\% rise in extent of gingival bleeding, attachment loss and pocket depth were associated with a $0.7,0.2$ and $0.6 \mathrm{mmHg}$ elevation in SBP respectively. Quite unexpectedly, the fully adjusted model of the study revealed a consistent association of SBP and hypertension only with gingival bleeding, a marker of acute periodontal inflammation. Moreover, only in men was there a clear linear trend between periodontitis severity and hypertension risk [10]. However, the advantage of our study is the definition of periodontitis case and the comparison of between all levels of periodontitis with blood pressure and hypertension. A casecontrol study from Brazil showed that arterial hypertension was significantly associated with severe chronic periodontitis and generalized chronic periodontitis, and the OR for them were 4.04 and 2.18, respectively [26]. Periodontitis is related with increased BP and WBC count [27]. The WBC count, SBP, and SBP were higher in subjects with periodontitis than in subjects without periodontitis, both at baseline and follow-up. Another study also showed that there was an association between DBP and prevalent deep periodontal pockets.

Although the causal relationship between periodontitis and hypertension may be difficult to prove, there are several potential pathophysiologic mechanisms that could explain the association between periodontitis and hypertension. 1) Inflammatory and Biologic Pathways: Population studies and experimental data have implicated diverse inflammatory mediators in the development of hypertension $[28,29]$. Many researches showed that inflammatory markers were found raised in periodontitis patients [30-32]. The possible inflammatory factors, which have been observed, consist of WBC, CRP, IL-6, TNF-a, E-selectin, vWf, PAI-1, and fibrinogen. 2) Endothelial Dysfunction: Periodontal disease may contribute to endothelial dysfunction, and management of oral health could improve endothelial function [33]. Periodontitis is associated with endothelial dysfunction in subjects with hypertension, through a decrease in NO bioavailability and that systemic inflammation may be a cause of endothelial dysfunction leading to raised blood pressure [34]. The increase of other endothelial factors was also observed in several studies $[12,35,36] .3)$ Oxidative Stress:
An imbalance between peroxidants and antioxidants results in oxidative stress, which causes the development of hypertension [37]. Another study showed that periodontitis-induced lipid peroxidation in the aorta may be involved in early atherosclerosis [38]. 4) Local bacteria, Bacteremia and Immune Response: Periodontal pathogens are able to invade gingival tissues and promote transient bacteremia [39]. Periodontal microbes may invade the arterial wall and colonize atherosclerotic plaques [40], which could result in raised blood pressure. 5) Interaction Among Insulin Resistance, Diabetes, Metabolic Syndrome: there was evidence to support the relationships between metabolic disturbances and periodontitis, where insulin resistance play a central role [41]. Recent meta-analysis showed evidence for an association between metabolic syndrome and periodontitis [42]. Furthermore, individuals with metabolic syndrome are more likely to suffer from periodontitis than individuals without this condition. Insulin resistance and oxidative stress seem to be common elements present in metabolic syndrome, periodontitis, cardiovascular disease and hypertension $[43,44]$.

On the contrary, there are also some evidences to object to correlation between periodontitis and hypertension. An analysis of data derived from the national Health 2000 Survey in Finland showed that both the number of teeth with deepened and the number of bleeding sextants were not consistently associated with hypertension after adjusting for confounding factors [45]. A follow-up study about the Health Professionals indicated that an association between periodontal disease measures and incident hypertension in middle-aged men was not observed [46]. Furthermore, a recent systematic review concluded that there was still no high-quality evidence that periodontal disease was associated with hypertension [47]. The associations found were from observational studies, but randomized trials did not show convincingly that periodontal therapy has a beneficial effect on hypertension.

We realized that our study is based on anepidemiological investigation and therefore cannot indicate or support a causal relationship between periodontitis and blood pressure or hypertension. Indeed, reverse causality whereby hypertension leads to periodontitis cannot be excluded. We have no data on risk factors for hypertension other than age and gender. Therefore, adjustment for other risk factors, such as diabetes, smoking, hyperlipidemia, and social status, could not be performed. It is obvious that conclusive evidence on the different mechanisms is beyond the remit of this study and randomized controlled trials are required for that purpose. Further studies are necessary to evaluate a possible causal relationship between periodontitis and arterial hypertension.

This was a cross-sectional study performed in a retrospective fashion and, therefore, suffered from inherent limitations. However, this could only impair the power of the study to disclose relationships but not induce false positive findings. 
The strength of the study is that specialists in periodontology diagnosed all subjects, and AL and PD are the main factors for categorizing the severity of periodontal disease. The results of our study were based on data from the National Health and Nutrition Examination Survey, which had a large sample and high population representation. In our study, we used clinically measured BP, both as a continuous variable and in a binary format in terms of hypertension. The definition of periodontitis and its severity was authoritative and commonly accepted. Furthermore, we adjusted for a number of risk factors for blood pressure that referred to different characteristics (socio-demographic, behavioral, physiological, chronic conditions). Apart from the statistical significance of the association, attention should also be paid to its magnitude and clinical significance.

\section{Conclusion}

Gender, age, military status, and marital status are possible risk factors for both periodontitis, blood pressure, and hypertension. We did observe the relationship between periodontitis, blood pressure and hypertension. Further studies are needed to identify confounding factors and evaluate causal relationship between periodontitis and hypertension.

\section{Acknowledgements}

The 2001-2002 NHANES Oral Health Component is a collaborative effort between the National Institute of Dental and Craniofacial Research (NIDCR), the National Center for Chronic Disease Prevention and Health Promotion, Division of Oral Health (NCCDPHP/DOH), and the National Center for Health Statistics (NCHS). Funding: The work was supported by grant number (no.20170313) Hebei Health and Family Planning Commission.

\section{References}

1. Löe H, Anerud A, Boysen H, Morrison E (1986) Natural history of periodontal disease in man. Rapid, moderate and no AL in Sri Lankan laborers 14 to 46 years of age. J Clin Periodontol 13(5): 431-445.

2. Joshipura KJ, Wand HC, Merchant AT, Rimm EB (2004) Periodontal disease and biomarkers related to cardiovascular disease. J Dent Res 83(2): 151-155.

3. Sanz M, D’Aiuto F, Deanfield J, Fernandez-Avilés F (2010) European workshop in periodontal health and cardiovascular diseasescientific evidence on the association between periodontal and cardiovascular diseases: a review of the literature. Eur Heart J Suppl 12: p. 10.

4. Burt B (2005) Position paper: epidemiology of periodontal diseases. J Periodontol 76(8): 1406-1419.

5. Go AS, Mozaffarian D, Roger VL, Benjamin EJ, Berry JD, et al. (2013) Heart disease and stroke statistics--2013 update: a report from the American Heart Association. Circulation 127(1): p. e6-e245.

6. Kakar P, Lip GYH (2006) Towards understanding the aetiology and pathophysiology of human hypertension: where are we now? J Hum Hypertens 20(11): 833-836.

7. Felmeden DC, Lip GYH (2005) Endothelial function and its assessment. Expert Opin Investig Drugs 14(11): 1319-1336.

8. Yin R, Li H, Wu J, Lin W, Yang D, et al. (2007) Effects of alcohol consumption and other lifestyle behaviors on blood pressure for the middle-aged and elderly in the Guangxi Hei Yi Zhuang and Han populations. Alcohol 41(8): p. 541-550.

9. Niskanen L, Laaksonen DE, Nyyssönen K, Punnonen K, Valkonen VP, et al. (2004) Inflammation, abdominal obesity, and smoking as predictors of hypertension. Hypertension 44(6): 859-865.

10. Tsakos G, Sabbah W, Hingorani AD, Netuveli G, Donos N, et al. (2010) Is Periodontal Inflammation Associated with Raised Blood Pressure? Evidence From a National US Survey. J hypertens 28(12): 2386-2393.

11. Roy C Page, Eke PI (2007) Case Definitions for Use in Population-Based Surveillance of Periodontitis. Journal of periodontology 78(7 Supp): 1387-1399.

12. Fujioka D, Nakamura S, Yoshino H, Shinohara H, Shiba H, et al. (2003) Expression of endothelins and their receptors in cells from human periodontal tissues. J Periodontal Res 38(3): p. 269-275.

13. Sanz M, Marco Del Castillo A, Jepsen S, Gonzalez-Juanatey JR, D’Aiuto F, et al. (2020) Periodontitis and Cardiovascular Diseases. Consensus Report. Glob Heart 15(1): p. 1.

14. Dubey RK, Oparil S, Imthurn B, Jackson EK (2002) Sex hormones and hypertension. Cardiovasc Res 53(3): p. 688-708.

15. Sowers JR, Lester M (2000) Hypertension, hormones, and aging. J Lab Clin Med 135(5): p. 379-386.

16. Ouzounian JG, Elkayam U (2012) Physiologic changes during normal pregnancy and delivery. Cardiol Clin 30(3): p. 317-329.

17. Valdés G, Corthorn J (2011) Challenges posed to the maternal circulation by pregnancy. Integr Blood Press Control 4: p. 45-53.

18. Hunter S, Robson SC (1992) Adaptation of the maternal heart in pregnancy. Br Heart J 68(6): p. 540-543.

19. Meisel P, Kohlmann T, Nauck M, Biffar R, Kocher T (2017) Effect of body shape and inflammation on tooth loss in men and women. Clin Oral Investig 21(1): p. 183-190.

20. Susin C, Haas AN, Albandar JM (2014) Epidemiology and demographics of aggressive periodontitis. Periodontol 2000 65(1): p. 27-45.

21. Billings M, Holtfreter B, Papapanou PN, Mitnik GL, Kocher T, et al. (2018) Age-dependent distribution of periodontitis in two countries: Findings from NHANES 2009 to 2014 and SHIP-TREND 2008 to 2012. J Clin Periodontol 45 Suppl 20: p. S130-S148.

22. Abdellatif HM, Burt BA (1987) An epidemiological investigation into the relative importance of age and oral hygiene status as determinants of periodontitis. J Dent Res 66(1): p. 13-18.

23. Kim JK, Baker LA, Seirawan H, Crimmins EM (2012) Prevalence of oral health problems in U.S. adults, NHANES 1999-2004: exploring differences by age, education, and race/ethnicity. Spec Care Dentist 32(6): p. 234-241.

24. Horning GM, Hatch CL, Cohen ME (1992) Risk indicators for periodontitis in a military treatment population. J Periodontol 63(4): p. 297-302.

25. Persson GR, Persson RE, Hollender LG, Kiyak HA (2004) The impact of ethnicity, gender, and marital status on periodontal and systemic health of older subjects in the Trials to Enhance Elders' Teeth and Oral Health (TEETH). J Periodontol 75(6): p. 817-823.

26. Vidal F, Figueredo CMS, Cordovil I, Fischer RG (2011) Higher prevalence of periodontitis in patients with refractory arterial hypertension: a case-control study. Oral Diseases 17(6): 560-563.

27. Inoue K, Kobayashi Y, Hanamura H, Toyokawa S (2005) Association of periodontitis with increased white blood cell count and blood pressure. Blood Press 14(1): p. 53-58. 
28. Chrysohoou, C, Pitsavos C, Panagiotakos DB, Skoumas J, Stefanadis C (2004) Association between prehypertension status and inflammatory markers related to atherosclerotic disease: The ATTICA Study. Am J Hypertens 17(7): p. 568-573.

29. Sesso HD, Buring JE, Rifai N, Blake GJ, Gaziano JM, et al. (2003) $\mathrm{C}$-reactive protein and the risk of developing hypertension. Jama 290(22): p. 2945-2951.

30. Paraskevas S, Huizinga JD, Loos BG (2008) A systematic review and meta-analyses on $\mathrm{C}$-reactive protein in relation to periodontitis. J Clin Periodontol 35(4): p. 277-290.

31. Loos BG, Craandijk J, Hoek FJ, Wertheim-van Dillen PM, van der Velden U (2000) Elevation of systemic markers related to cardiovascular diseases in the peripheral blood of periodontitis patients. J Periodontol 71(10): p. 1528-1534.

32. Passoja A, Puijola I, Knuuttila M, Niemelä O, Karttunen R, et al. (2010) Serum levels of interleukin-10 and tumour necrosis factor- $\alpha$ in chronic periodontitis. J Clin Periodontol 37(10): p. 881-887.

33. Tonetti MS, D’Aiuto F, Nibali L, Donald A, Storry C, et al. (2007) Treatment of periodontitis and endothelial function. N Engl J Med 356(9): p. 911-920.

34. Higashi Y, Goto C, Jitsuiki D, Umemura T, Nishioka K, et al. (2008) Periodontal infection is associated with endothelial dysfunction in healthy subjects and hypertensive patients. Hypertension 51(2): p. 446-453.

35. Pradeep AR, Guruprasad CN, Swati P, Shikha C (2008) Crevicular fluid endothelin-1 levels in periodontal health and disease. J Periodontal Res 43(3): p. 275-278.

36. Tsioufis C, Thomopoulos C, Soldatos N, Syrseloudis D, Kasiakogias A et al. (2010) The conjoint detrimental effect of chronic periodontal disease and systemic inflammation on asymmetric dimethyl-arginine in untreated hypertensive subjects. Atherosclerosis 208(1): p. 258263.

37. Touyz RM (2004) Reactive oxygen species, vascular oxidative stress, and redox signaling in hypertension: what is the clinical significance? Hypertension 44(3): p. 248-252.
38. Ekuni D, Tomofuji T, Sanbe T, Irie K, Azuma T, et al. (2009) Periodontitisinduced lipid peroxidation in rat descending aorta is involved in the initiation of atherosclerosis. J Periodontal Res 44(4): p. 434-442.

39. Sandros J, Papapanou PN, Nannmark U, Dahlén G, et al. (1994) Porphyromonas gingivalis invades human pocket epithelium in vitro. J Periodontal Res 29(1): p. 62-69.

40. Kozarov EV, Dorn BR, Shelburne CE, Dunn Jr WA, Progulske-Fox A (2005) Human atherosclerotic plaque contains viable invasive Actinobacillus actinomycetemcomitans and Porphyromonas gingivalis. Arterioscler Thromb Vasc Biol 25(3): p. e17-e18.

41. Benguigui C, Bongard V, Ruidavets JB, Chamontin B, Sixou M, et al. (2010) Metabolic syndrome, insulin resistance, and periodontitis: a cross-sectional study in a middle-aged French population. J Clin Periodontol 37(7): p. 601-608.

42. Daudt LD, Musskopf ML, Mendez M, Remonti LLR, Leitão CB, et al. (2018) Association between metabolic syndrome and periodontitis: a systematic review and meta-analysis. Braz Oral Res 32: p. e35.

43. Grossi SG, Genco RJ (1998) Periodontal disease and diabetes mellitus: a two-way relationship. Ann Periodontol 3(1): p. 51-61.

44. Marchetti E, Monaco A, Procaccini L, Mummolo S, Gatto R, et al. (2012) Periodontal disease: the influence of metabolic syndrome. Nutr Metab (Lond) 9(1): p. 88.

45. Ollikainen E, Saxlin T, Tervonen T, Suominen AL, Knuuttila M, et al. (2014) Association Between Periodontal Condition and Hypertension in a Non-Smoking Population Aged 30-49 Years: Results of the Health 2000 Survey in Finland. J Clin periodontol 41(12): p. 1132-1138.

46. Rivas-Tumanyan S, Spiegelman D, Curhan GC, Forman JP, Joshipura KJ (2012) Periodontal Disease and Incidence of Hypertension in the Health Professionals Follow-Up Study. American journal of hypertension 25(7): p. 7.

47. Brignardello-Petersen R (2020) There is still no high-quality evidence that periodontitis is a risk factor for hypertension or that periodontal treatment has beneficial effects on blood pressure. J Am Dent Assoc 151(4): p. e31. 